\title{
Photocoagulation and diathermy in the treatment of proliferative sickle retinopathy
}

\author{
PATRICK I. GONDON AND GRAHAM R. SERJEANT \\ From the Eye Sickle Cell Clinic, St. Thomas's Hospital, London, and the Medical Research Council, \\ Epidemiology Unit, University of West Indies, Kingston, Jamaica
}

Small vessel obstruction, characteristic of sickle cell disease, may lead to retinal ischaemia and the development of retinitis proliferans (RP). The frequency of this lesion varies with the genotype of sickle cell disease; it occurred in 2.6 per cent. of 76 patients with homozygous sickle cell disease (SS disease), I 4 per cent. of fifty patients with sickle cell-thalassaemia (S-thal), and in 32.8 per cent. patients with sickle cell-haemoglobin $\mathrm{C}$ disease (SC disease) in three surveys in Jamaica (Condon and Serjeant, I972a,b,c).

Evidence that retinal vascular disease and RP are progressive (Goldberg, I97 Ia) has been supported by a recent $2 \frac{1}{2}$-year longitudinal study in Jamaica (Condon and Serjeant, 1974, 1975) and has justified attempts to treat these lesions in the hope of preventing this progression. Hannon (1956) treated RP in two cases of SC disease by scleral surface diathermy and found good immediate results, although long term follow-up was not available. Goldberg (197 Ib) used xenon arc photocoagulation in fifteen patients with various sickle cell disorders and succeeded in occluding vasculature in 74 per cent. of RP lesions in short-term studies, but the long-term effects remain unknown. Despite the poor effect of Argon laser photocoagulation in the closure of normal human retinal arterioles (Apple, Goldberg, and Wyhinny, 1973), Goldberg and Acacio (1973) reported successful occlusion of RP lesions by Argon laser photocoagulation in sixteen patients with SG but the I4 per cent. incidence of immediate vitreous haemorrhage was three times that found in the xenon arc study. The present study was undertaken to assess the efficiency of a small portable xenon arc photocoagulator (O’Malley Log II Photocoagulator) and of a portable diathermy machine (Manchester) in these lesions. This report presents the technique and preliminary results of therapy.

\section{Glinical material and methods}

Patients were collected from two sources. Cases I to 7 (Table I, pp. 652 to 655) came from the retinal clinic of the Royal Eye Hospital and from the sickle cell clinic of St. Thomas' Hospital, London, during the period between November, 1969, and January, 1973. Cases 8 to 44 attended the sickle cell clinic at the University Hospital of the West Indies and were treated between April and June, 1973.

Received for publication September 27, 1973

Address for reprints: P. I. Condon, F.R.C.S., South-East Area Health Board Regional Eye Department, Ardkeen Hospital, Waterford, Ireland

This study was supported by the Wellcome Trust, the South-East Metropolitan Regional Hospital Board and the Medical Research Council 

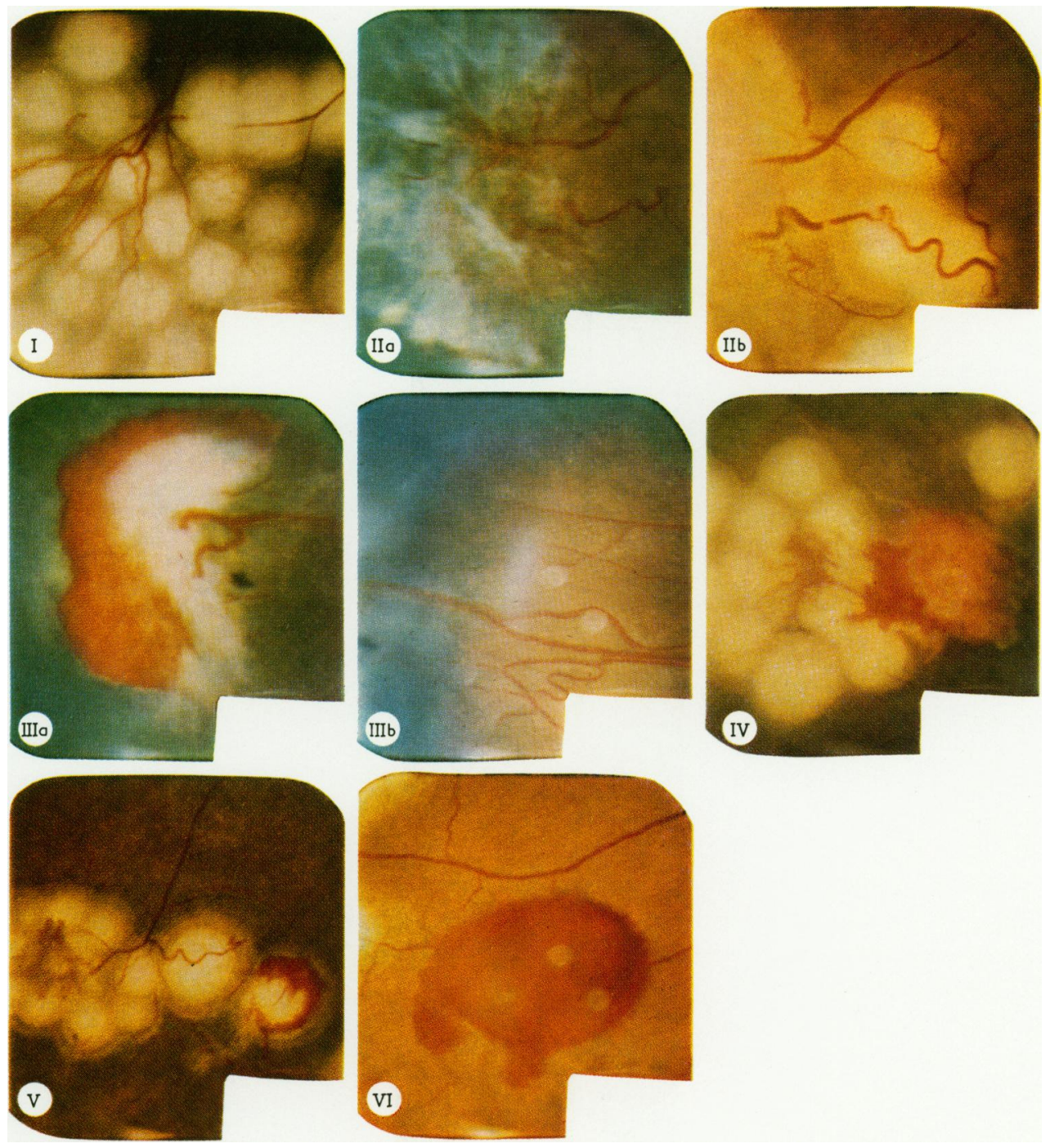

PLATE I Case 33. Photocoagulation of RP lesion. Major feeder arteriole totally occluded

PLATE II $(a, b)$ Case I2. Large elevated sea-fan lesion with vitreo-retinal traction before and after treatment. Photocoagulation burns to large feeder arterioles on the left and diathermy reaction to sea-fan on right of $(b)$

PLATE III $(a, b)$ Case 36 . Typical haemorrhagic sea-fan lesion with large feeder retinal vessels flat on retinal surface in the one good eye of this patient

PLATE IV Case IO. Preretinal haemorrhage from accidental photocoagulation of draining retinal vein from $R P$ lesion

PLATE V Case 16 . Localized retinal haemorrhage into photocoagulation burn of small feeder arteriole PLATE VI Case I2. Preretinal haemorrhage related to feeder arteriole posterior to diathermized area 
The 44 patients ( 28 male; 6 female) included 34 cases of SC, six of S-thal, and four of SS disease. The criteria for the haematological diagnosis were as described by Serjeant and Serjeant (1972).

The methods of ophthalmological examination have been described by Condon and Serjeant (1972a) and the diagnosis of RP or sea-fan lesions was based on the ophthalmological appearance and on the leakage of intravenously administered fluorescein dye. When lack of cooperation or the extreme peripheral location of the lesion to be treated did not allow retinal photography, leakage of dye was confirmed by observation through an indirect ophthalmoscope fitted with appropriate filters (Keith, 1968). In the 44 patients, RP occurred unilaterally in I I and bilaterally in 33; in each eye, the extent of peripheral retinal involvement was assessed in degrees and the numbers of RP lesions and their feeding arterioles were recorded. In the patients with bilateral disease, the eye with the most extensive involvement was treated, the other being left as a control. If, however, vision was reduced to $6 / 60$ or less in the worse eye, the better eye was treated. Treatment was applied to all lesions in the treated eye.

The aim of therapy was to render RP lesions avascular so that fluorescein leakage into the vitreous no longer occurred. For the purpose of this report, this was defined as a 'therapeutic cure'. The choice of patients for one or other of the specific forms of therapy depended on the type of RP present, the relation of the new vessels to the retinal surface, and the presence of vitreo-retinal traction. Photo coagulation was used in lesions which lay on the retinal surface with or without a minimum degree of vitreo-retinal traction but without any form of secondary schisis or retinal detachment (Plate I; Figs I $a, b, c)$. Diathermy was reserved for the advanced type of RP with fibrosis of the vitreous,
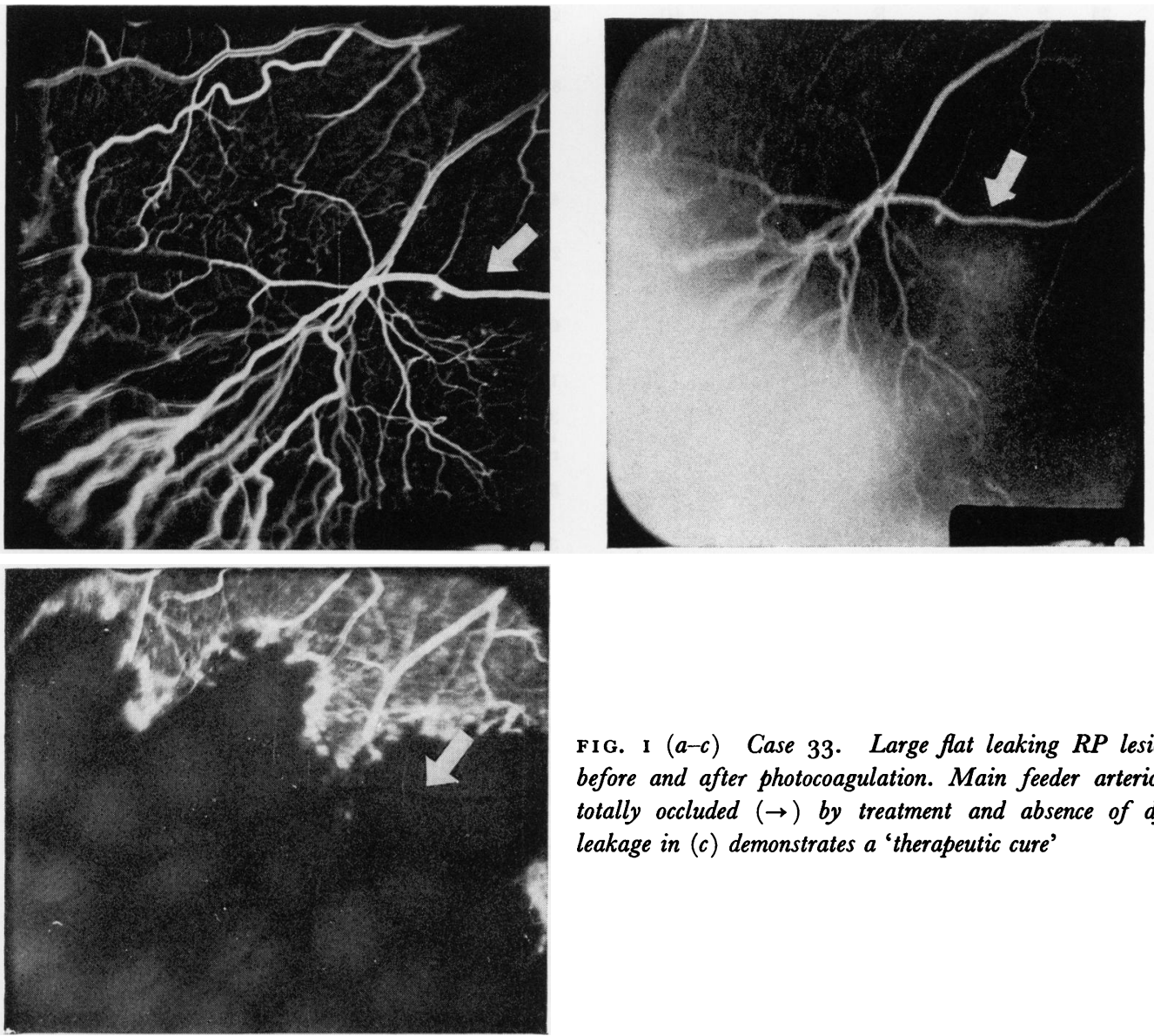

FIG. I $(a-c)$ Case 33. Large flat leaking RP lesion before and after photocoagulation. Main feeder arteriole totally occluded $(\rightarrow)$ by treatment and absence of dye leakage in (c) demonstrates a 'therapeutic cure' 
Table I Results of treating 44 patients by photocoagulation and diathermy

\begin{tabular}{|c|c|c|c|c|c|c|c|c|c|c|}
\hline \multirow{2}{*}{$\begin{array}{l}\text { Patient } \\
\text { no. }\end{array}$} & \multirow[b]{2}{*}{$\begin{array}{l}A g e \\
(y r s)\end{array}$} & \multirow[b]{2}{*}{ Sex } & \multirow[b]{2}{*}{$\begin{array}{l}\text { Haemo- } \\
\text { globin }\end{array}$} & \multirow[b]{2}{*}{ Eye } & \multirow{2}{*}{$\begin{array}{l}\text { Presenting } \\
\text { symptom }\end{array}$} & \multicolumn{2}{|c|}{ No. of $R P$ lesions treated } & \multirow{2}{*}{ 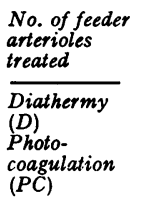 } & \multirow{2}{*}{$\begin{array}{l}\text { Associated } \\
\text { Traction }(T) \\
\text { Retinoschisis } \\
\text { (TS) } \\
\text { Retinal } \\
\text { detachment } \\
(T R D) \\
\text { Tear } \pm\end{array}$} & \multirow{2}{*}{$\begin{array}{l}\text { Area of retina treated }\left(^{\circ}\right) \\
\text { Diathermy } \\
\text { Photocoagulation includes } \\
R P, T S, T R D, \text { and } \\
\text { border zone retina with } \\
\text { new vessels and } A-V \\
\text { shunts }\end{array}$} \\
\hline & & & & & & $\begin{array}{l}\text { Diathermy } \\
R x \text { nos. }\end{array}$ & $\begin{array}{l}\begin{array}{l}\text { Photo- } \\
\text { coagulation }\end{array} \\
\text { Rx nos. }\end{array}$ & & & \\
\hline 1 & 32 & $\mathbf{F}$ & $\mathrm{SC}$ & $\mathbf{R}$ & \multirow{2}{*}{$\begin{array}{l}\text { Vitreous } \\
\text { haemorrhage } \\
-\end{array}$} & $2 / 1$ & - & 2 (D) & \multirow{2}{*}{$\begin{array}{l}1 \text { TRD } \\
+ \text { Tear } \\
1 \text { T }\end{array}$} & \multirow{2}{*}{$\begin{array}{l}100^{\circ}(\mathrm{D}) \\
\text { under lateral rectus } \\
120^{\circ} \text { (D) } \\
\text { under lateral rectus }\end{array}$} \\
\hline 2 & 36 & $\mathrm{~F}$ & $\mathrm{SC}$ & $\mathbf{R}$ & & $2 / 1$ & - & $2(\mathrm{D})$ & & \\
\hline 3 & 34 & $\mathbf{M}$ & $\mathrm{SC}$ & $\mathrm{L}$ & \multirow{3}{*}{$\begin{array}{l}\text { Vitreous } \\
\text { haemorrhage } \\
\text { Vitreous } \\
\text { haemorrhage } \\
-\end{array}$} & $4 / 1$ & - & 7 (D) & 1 TRD & \multirow{4}{*}{$\begin{array}{l}90^{\circ}(\mathrm{D}) \\
\text { under lateral rectus } \\
120^{\circ}(\mathrm{D}) \\
\text { under medial rectus } \\
90^{\circ}(\mathrm{D}) \\
\text { under lateral rectus } \\
90^{\circ} \text { (D) } \\
\text { under lateral rectus }\end{array}$} \\
\hline 4 & 30 & $\mathrm{~F}$ & $\mathrm{SC}$ & $\mathbf{L}$ & & $3 / 2$ & - & 6 (D) & 1 TS & \\
\hline 5 & 33 & $\mathbf{M}$ & $\mathrm{SC}$ & $\mathrm{L}$ & & $5 / 1$ & - & 5 (D) & $1 \mathrm{~T}$ & \\
\hline 6 & 36 & $\mathbf{M}$ & $\mathrm{SC}$ & $\mathbf{R}$ & - & $3 / 1$ & - & 5 (D) & $1 \mathrm{~T}$ & \\
\hline 7 & 24 & $\mathbf{F}$ & $\mathrm{SC}$ & $\mathrm{R}$ & - & $\begin{array}{l}1 / 1 \\
\text { Previous } \\
\text { failed } \\
\text { PC }\end{array}$ & - & 2 (D) & $1 \mathrm{~T}$ & $\begin{array}{l}90^{\circ}(\mathrm{D}) \\
\text { under lateral rectus }\end{array}$ \\
\hline 8 & 15 & M & $\mathrm{SC}$ & $\mathrm{L}$ & - & $1 / 1$ & - & 3 (D) & 1 TRD & $30^{\circ}(\mathrm{D})$ \\
\hline 9 & 25 & $\mathbf{F}$ & $\mathrm{SC}$ & $\mathrm{R}$ & - & $4 / 1$ & $2 / 1$ & $\begin{array}{l}4 \stackrel{(\mathrm{D})}{4}) \\
(\mathrm{PC})\end{array}$ & $\begin{array}{l}3 \text { TRD } \\
- \text { Tear }\end{array}$ & $\begin{array}{l}180^{\circ}(\mathrm{D}) \\
10^{\circ}(\mathrm{PC})\end{array}$ \\
\hline 10 & 22 & M & $\begin{array}{l}\text { SC } \\
\text { Plate IV }\end{array}$ & $\mathrm{L}$ & - & $1 / 1$ & $5 / 1$ & $\begin{array}{l}5(\mathrm{D}) \\
11(\mathrm{PC})\end{array}$ & $1 \mathrm{TS}$ & $\begin{array}{l}75^{\circ}(\mathrm{D}) \\
\text { under lateral rectus } \\
120^{\circ}(\mathrm{PC})\end{array}$ \\
\hline 11 & 28 & M & $\mathrm{sc}$ & $\mathbf{R}$ & $\begin{array}{l}\text { Vitreous } \\
\text { haemorrhage } \\
\mathrm{R}\end{array}$ & $1 / 1$ & $4 / 1$ & $\begin{array}{l}2 \text { (D) } \\
5 \text { (PC) }\end{array}$ & $1 \mathrm{TS}$ & $\begin{array}{l}30^{\circ}(\mathrm{D}) \\
60^{\circ}(\mathrm{PC})\end{array}$ \\
\hline 12 & 29 & M & $\begin{array}{l}\text { S-Thal } \\
\text { Plate II } \\
\text { Fig. } 2 \\
\text { Plate VI }\end{array}$ & $\mathrm{L}$ & - & $1 / 1$ & $2 / 1$ & $\begin{array}{l}5 \text { (D) } \\
4(\mathrm{PC})\end{array}$ & $1 \mathrm{TS}$ & $\begin{array}{l}90^{\circ}(\mathrm{D}) \\
\text { under lateral rectus } \\
10^{\circ}(\mathrm{PC})\end{array}$ \\
\hline 13 & 54 & $\mathrm{~F}$ & SS & $\mathrm{R}$ & - & $1 / 1$ & $6 / 1$ & $\begin{array}{l}2 \text { (D) } \\
9 \text { (PC) }\end{array}$ & $1 \mathrm{~T}$ & $\begin{array}{l}30^{\circ}(\mathrm{D}) \\
120^{\circ}(\mathrm{PC})\end{array}$ \\
\hline 14 & 15 & M & $\mathrm{SC}$ & $\mathrm{R}$ & - & - & $1 / 1$ & $2(\mathrm{PC})$ & - & $15^{\circ}(\mathrm{PC})$ \\
\hline 15 & 15 & $\mathrm{~F}$ & $\mathrm{sc}$ & $\mathrm{R}$ & - & - & $\begin{array}{l}3 / 1 \\
1 \text { RP still } \\
\text { remains }\end{array}$ & $9(\mathrm{PC})$ & - & $120^{\circ}(\mathrm{PC})$ \\
\hline 16 & 36 & $\mathbf{M}$ & $\begin{array}{l}\mathrm{SC} \\
\text { Plate V }\end{array}$ & $\mathrm{L}$ & - & - & $5 / 1$ & $11(\mathrm{PC})$ & - & $100^{\circ}(\mathrm{PC})$ \\
\hline 17 & 25 & M & $\mathrm{sc}$ & $\mathrm{R}$ & - & - & $3 / 1$ & $7(\mathrm{PC})$ & - & $90^{\circ}(\mathrm{PC})$ \\
\hline 18 & 62 & $\mathrm{~F}$ & $\mathrm{sc}$ & $\mathrm{R}$ & - & - & $2 / 1$ & $3(\mathrm{PC})$ & - & $60^{\circ}(\mathrm{PC})$ \\
\hline 19 & 50 & $\mathrm{~F}$ & $\mathrm{sc}$ & $\mathrm{L}$ & $\begin{array}{l}\text { Vitreous } \\
\text { haemorrhage } \\
\text { Posterior ciliary } \\
\text { artery } \\
\text { occlusion } \\
\mathrm{R}\end{array}$ & - & $6 / 1$ & $2(\mathrm{PC})$ & - & $90^{\circ}(\mathrm{PC})$ \\
\hline 20 & 20 & M & $\mathrm{SC}$ & $\mathbf{L}$ & - & - & $1 / 1$ & $1(\mathrm{PC})$ & - & $100^{\circ}(\mathrm{PC})$ \\
\hline 21 & 37 & $\mathrm{~F}$ & $\mathrm{SC}$ & $\mathbf{R}$ & - & - & $1 / 1$ & $2(\mathrm{PC})$ & - & $15^{\circ}(\mathrm{PC})$ \\
\hline 22 & 28 & $\mathbf{M}$ & $\mathrm{SC}$ & $\mathbf{R}$ & $\begin{array}{l}\text { Vitreous } \\
\text { haemorrhage }\end{array}$ & - & $4 / 1$ & $5(\mathrm{PC})$ & - & $90^{\circ}(\mathrm{PC})$ \\
\hline 23 & 46 & M & $\mathrm{sc}$ & $\mathrm{L}$ & - & - & $2 / 1$ & $2(\mathrm{PC})$ & - & $45^{\circ}(\mathrm{PC})$ \\
\hline 24 & 30 & M & $\mathrm{sC}$ & $\mathbf{R}$ & $\begin{array}{l}\text { Retinal } \\
\text { detachment } \\
\text { L }\end{array}$ & - & $10 / 1$ & $15(\mathrm{PC})$ & - & $300^{\circ}(\mathrm{PC})$ \\
\hline 25 & 18 & M & $\mathrm{sc}$ & $\mathrm{L}$ & - & - & $1 / 1$ & $3(\mathrm{PC})$ & - & $270^{\circ}(\mathrm{PC})$ \\
\hline 26 & 28 & $\mathrm{~F}$ & $\mathrm{sc}$ & L & - & - & $1 / 1$ & $1(\mathrm{PC})$ & - & $120^{\circ}(\mathrm{PC})$ \\
\hline
\end{tabular}




\begin{tabular}{|c|c|c|}
\hline \multirow{2}{*}{$\begin{array}{l}\text { Treatment details } \\
\text { Diathermy (ma/sec.) } \\
\text { *Photocoagulation No.; } \\
\text { Intensity (I); Size (S); } \\
\text { Duration (sec.) (D) }\end{array}$} & \multicolumn{2}{|c|}{ Visual acuity } \\
\hline & $\begin{array}{l}\text { Before } \\
\text {; treatment }\end{array}$ & $\begin{array}{l}\text { After } \\
\text { treatment } \\
\text { (follow-up) }\end{array}$ \\
\hline D: $70 \mathrm{ma} / 6 \mathrm{sec}$. & $6 / 9$ & \multirow{2}{*}{$\begin{array}{l}6 / 9 \\
(40 \mathrm{wks}) \\
6 / 6 \\
(2 \mathrm{yrs})\end{array}$} \\
\hline D: $70 \mathrm{ma} / 7 \mathrm{sec}$. & $6 / 6$ & \\
\hline D: $70 \mathrm{ma} / 8 \mathrm{sec}$ & $6 / 9$ & \multirow{4}{*}{$\begin{array}{l}6 / 6 \\
(24 \mathrm{wks}) \\
6 / 6 \\
(18 \mathrm{mths}) \\
6 / 9 \\
(18 \mathrm{mths}) \\
6 / 9 \\
(2 \mathrm{yrs})\end{array}$} \\
\hline D: $75 \mathrm{ma} / 5 \mathrm{sec}$ & $6 / 9$ & \\
\hline D: $60 \mathrm{ma} / 7 \mathrm{sec}$. & $6 / 9$ & \\
\hline D: $70 \mathrm{ma} / 8 \mathrm{sec}$. & $6 / 9$ & \\
\hline
\end{tabular}

\begin{tabular}{|c|c|c|c|}
\hline $\mathrm{D}: 60 \mathrm{ma} / 7 \mathrm{sec}$. & $6 / 6$ & $\begin{array}{l}6 / 36 \\
(2 \mathrm{yrs})\end{array}$ & $\mathrm{S}^{\text {Leakage - }}$ \\
\hline D: $80 \mathrm{ma} / 5 \mathrm{sec}$. & $6 / 6$ & $\begin{array}{l}6 / 6 \\
(4 \mathrm{wks})\end{array}$ & Leakage - \\
\hline $\begin{array}{l}\text { D: } 70 \mathrm{ma} / 6 \mathrm{sec} \\
\text { PC: } 10 ; 8 \text { (I); } \\
4.5 \text { (S); } 1.5 \text { (D) }\end{array}$ & $6 / 6$ & $\begin{array}{l}6 / 9 \\
\text { ( } 4 \text { wks) } \\
\text { vitreous haze }\end{array}$ & $\begin{array}{l}\text { Leakage - } \\
\text { RD }\end{array}$ \\
\hline D: $70 \mathrm{ma} / 6 \mathrm{sec}$. & $6 / 6$ & $6 / 6$ & L.eakage \\
\hline
\end{tabular}

\begin{tabular}{|c|c|c|c|}
\hline $\begin{array}{l}\text { PC: } 45,7 \text { (I); } \\
3 \text { (S), } 1.5 \text { (D) }\end{array}$ & $6 / 6$ & \multirow{3}{*}{$\begin{array}{l}6 / 6 \\
(8 \mathrm{wks}) \\
6 / 6 \\
\text { (8 wks) } \\
6 / 6 \\
\text { (7 wks) }\end{array}$} & Leakage - \\
\hline $\begin{array}{l}\mathrm{PC}: 25,5(\mathrm{I}) \\
4.5 \text { (S) } 1.5(\mathrm{D})\end{array}$ & $6 / 6$ & & Leakage - \\
\hline $\begin{array}{l}\text { PC: } 34 ; 8 \text { (I); } \\
6 \text { (S); } 2.5 \text { (D) } \\
\text { Vitreous haze }\end{array}$ & $6 / 6$ & & Leakage - \\
\hline $\begin{array}{l}\text { PC: } 50,7 \text { (I), } \\
3 \text { (S), } 1.5 \text { (D) }\end{array}$ & $6 / 6$ & \multirow{2}{*}{$\begin{array}{l}6 / 6 \\
(5 \mathrm{wks}) \\
6 / 6 \\
\text { (4 wks) }\end{array}$} & Leakage - \\
\hline $\begin{array}{l}\mathrm{PC}: 169,9(\mathrm{I}) \\
4.5(\mathrm{~S}), 3 \text { (D) } \\
\text { Large arteriole }\end{array}$ & $6 / 6$ & & $\begin{array}{l}\text { Leakage - } \\
\text { (9 RPs) } \\
\text { Leakage + }\end{array}$ \\
\hline $\begin{array}{l}\text { PC: } 120,8(\mathrm{I}) \\
4 \cdot 5(\mathrm{~S}), 2(\mathrm{D})\end{array}$ & $6 / 6$ & \multirow{2}{*}{$\begin{array}{l}6 / 6 \\
(2 \mathrm{wks}) \\
6 / 6 \\
(2 \mathrm{wks})\end{array}$} & Leakage - \\
\hline $\begin{array}{l}\mathrm{PC}: 38,8(\mathrm{I}), \\
4.5(\mathrm{~S}), 2.5 \\
\text { (D) }\end{array}$ & $6 / 6$ & & Leakage - \\
\hline
\end{tabular}

Complications

Anterior uveitis (6 wks)

Anterior uveitis (6 wks)

1 preretinal haemorrhage behind

treated area

Anterior and posterior uveitis (4 wks)

Anterior uveitis (4 wks)

Anterior uveitis (4 wks)

Anterior and posterior uveitis (4 wks)

1 retinal haemorrhage optic disc

2 retinal haemorrhages untreated A- $V$ fistulae

1 small localized vitreous

haemorrhage in treated RP

Posterior ciliary artery occlusion 12

wks after treatment-possibly unrelated

Anterior uveitis (1 wk)

Anterior segment ischaemia $2 \mathrm{mths}$ after treatment

4 preretinal haemorrhages behind treated area

Anterior uveitis ( $4 \mathrm{wks}$ )

1 large preretinal haemorrhage due to inadvertent coagulation of draining
ina pretinal haemorrage due to vein of RP lesion

3 preretinal and 1 deep retinal haemorrhage distal to treated area Anterior uveitis 2 wks

Anterior uveitis 4 wks

3 preretinal haemorrhages posterior, and 2 distal to treated area

Anterior uveitis 2 wks

2 retinal haemorrhages in coagulation burns

1 retinal haemorrhage in coagulation burn

Did not attend again for further treatment 
Table I (continued)

\begin{tabular}{|c|c|c|c|c|c|c|c|c|c|c|}
\hline \multirow[b]{2}{*}{$\begin{array}{l}\text { Patient } \\
\text { no. }\end{array}$} & \multirow[b]{2}{*}{$\begin{array}{r}\text { Age } \\
(y r s)\end{array}$} & \multirow[b]{2}{*}{ Sex } & \multirow[b]{2}{*}{$\begin{array}{l}\text { Haemo- } \\
\text { globin }\end{array}$} & \multirow[b]{2}{*}{ Eye } & \multirow[b]{2}{*}{$\begin{array}{l}\text { Presenting } \\
\text { symptom }\end{array}$} & \multicolumn{2}{|c|}{ No. of $R P$ lesions treated } & \multirow{2}{*}{$\begin{array}{l}\begin{array}{l}\text { No. of feeder } \\
\text { arterioles } \\
\text { treated }\end{array} \\
\text { Diathermy } \\
(D) \\
\text { Photo- } \\
\text { coagulation } \\
(P C)\end{array}$} & \multirow{2}{*}{$\begin{array}{l}\text { Associated } \\
\text { Traction }(T) \\
\text { Retinoschisis } \\
\text { (TS) } \\
\text { Retinal } \\
\text { detachment } \\
(T R D) \\
\text { Tear } \pm \\
\end{array}$} & \multirow{2}{*}{$\begin{array}{l}\text { Area of retina treated }\left(^{\circ}\right) \\
\text { Diathermy } \\
\text { Photocoagulation includes } \\
R P, T S, T R D, \text { and } \\
\text { border zone retina with } \\
\text { new vessels and } A V \\
\text { shunts }\end{array}$} \\
\hline & & & & & & $\begin{array}{l}\text { Diathermy } \\
R x \text { nos. }\end{array}$ & $\begin{array}{l}\text { Photo- } \\
\text { coagulation } \\
\text { Rx nos. }\end{array}$ & & & \\
\hline 27 & 22 & $\mathbf{M}$ & $\mathrm{SC}$ & $\overline{\mathrm{R}}$ & - & - & $7 / 1$ & $13(\mathrm{PC})$ & $\overline{-}$ & $220^{\circ}(\mathrm{PC})$ \\
\hline 28 & 19 & $\mathbf{M}$ & $\mathrm{sc}$ & $L$ & - & & $2 / 1$ & $2(\mathrm{PC})$ & - & $90^{\circ}(\mathrm{PC})$ \\
\hline 29 & 13 & $\mathrm{~F}$ & $\mathrm{sc}$ & $\mathbf{R}$ & - & - & $3 / 1$ & $3(\mathrm{PC})$ & - & $150^{\circ}(\mathrm{PC})$ \\
\hline 30 & 45 & $\mathbf{M}$ & S-Tha & $\mathbf{R}$ & $\begin{array}{l}\text { Vitreous } \\
\text { haemorrhage }\end{array}$ & - & $6 / 1$ & $6(\mathrm{PC})$ & - & $150^{\circ}(\mathrm{PC})$ \\
\hline 31 & 27 & $\mathbf{M}$ & S-Thal & $L$ & - & - & $5 / 1$ & $5(\mathrm{PC})$ & - & $90^{\circ}(\mathrm{PC})$ \\
\hline 32 & 33 & $\mathbf{M}$ & S-Thal & L & - & - & $2 / 1$ & $2(\mathrm{PC})$ & - & $120^{\circ}(\mathrm{PC})$ \\
\hline 33 & 18 & $\mathbf{M}$ & $\begin{array}{l}\text { S-Thal } \\
\text { Plate I }\end{array}$ & $\mathbf{R}$ & - & - & $2 / 1$ & $7(\mathrm{PC})$ & - & $150^{\circ}(\mathrm{PC})$ \\
\hline 34 & 32 & $\mathbf{F}$ & SS & $\mathrm{R}$ & - & - & $1 / 1$ & $3(\mathrm{PC})$ & - & $30^{\circ}(\mathrm{PC})$ \\
\hline 35 & 36 & $\mathbf{M}$ & ss & $\mathbf{R}$ & - & - & $1 / 1$ & $3(\mathrm{PC})$ & - & $45^{\circ}(\mathrm{PC})$ \\
\hline 36 & 24 & $\mathrm{~F}$ & $\begin{array}{l}\text { SC } \\
\text { Plate III } \\
\text { Figs } 3, \mathbf{4}\end{array}$ & $\mathrm{L}$ & $\begin{array}{l}\text { Retinal } \\
\text { detachment } \\
\text { R }\end{array}$ & - & $4 / 2$ & $13(\mathrm{PC})$ & - & $240^{\circ}(\mathrm{PC})$ \\
\hline 37 & 31 & $\mathbf{M}$ & $\mathrm{SC}$ & L & $\begin{array}{l}\text { Vitreous } \\
\text { haemorrhage } \\
\text { and Posterior } \\
\text { ciliary artery } \\
\text { occlusion } R\end{array}$ & - & $8 / 2$ & $15(\mathrm{PC}$ & - & $270^{\circ}(\mathrm{PC})$ \\
\hline 38 & 36 & $\mathbf{M}$ & sc & $\mathbf{R}$ & $\begin{array}{l}\text { Vitreous } \\
\text { haemorrhage } \\
R\end{array}$ & - & $6 / 2$ & $15(\mathrm{PC})$ & - & $240^{\circ}(\mathrm{PC})$ \\
\hline 39 & 51 & $\mathbf{M}$ & $\mathrm{sc}$ & $\mathbf{R}$ & - & - & $\begin{array}{l}8 / 2 \\
2 \text { RPs still } \\
\text { remain } \\
\text { Asteroid } \\
\text { hyalitis }\end{array}$ & $11(\mathrm{PC})$ & - & $210^{\circ}(\mathrm{PC})$ \\
\hline 40 & 62 & $\mathbf{M}$ & S-Thal & L & - & - & $\begin{array}{l}4 / 2 \\
1 \mathrm{RP} \text { still } \\
\text { remains } \\
\text { Pterygium } \\
\text { corneal } \\
\text { distortion }\end{array}$ & $4(\mathrm{PC})$ & - & $100^{\circ}(\mathrm{PC}$ \\
\hline 41 & $\$ 3$ & $\mathbf{F}$ & $\mathrm{SC}$ & L & - & - & $\begin{array}{l}3 / 2 \\
\text { First treat- } \\
\text { ment not } \\
\text { adequate }\end{array}$ & $4(\mathrm{PC})$ & - & $90^{\circ}(\mathrm{PC})$ \\
\hline 42 & 27 & $\mathbf{M I}$ & $\begin{array}{l}\text { SC } \\
\text { Fig. } 5\end{array}$ & $\mathbf{R}$ & Wo & - & $\begin{array}{l}7 / 2 \\
\text { First treat- } \\
\text { ment not } \\
\text { adequate } \\
\text { Feeder } \\
\text { arteriole } \\
\text { missed }\end{array}$ & $11(\mathrm{PC})$ & - & $210^{\circ}(\mathrm{PC})$ \\
\hline 43 & $2 ! 1$ & F & $\mathrm{SC}$ & L & $\begin{array}{l}\text { Vitreous } \\
\text { haemorrhage }\end{array}$ & - & $\begin{array}{l}6 / 2 \\
1 \text { RP still } \\
\text { remains } \\
\text { Vitreous } \\
\text { opacity }\end{array}$ & $8(\mathrm{PC})$ & - & $90^{\circ}(\mathrm{PC})$ \\
\hline 44 & 19 & M & SS & L & - & - & $\begin{array}{l}3 / 2 \\
\text { First treat- } \\
\text { ment not } \\
\text { adequate }\end{array}$ & $9(\mathrm{PC})$ & - & $120^{\circ}(\mathrm{PC})$ \\
\hline
\end{tabular}

preretinal location of the feeder arterioles secondary to vitreo-retinal traction, and the presence or absence of an associated localized retinal schisis or detachment (Plate II $a$; Fig. 2a). Standard retinal detachment procedures (silastic plomb application and encirclement operations) were used only if the retinal detachment extended beyond the area of traction exerted by the lesion and if a retinal tear was present. All patients were examined by fluorescein angiography before and after treatment.

\section{Photocoagulation technique}

Feeding arterioles of the RP lesions, located by fluorescein angiography before treatment, were confirmed by using the coagulator as an ophthalmoscope at the time of treatment. Photocoagulation, using intensities varying between 5 and 10 , burn size between 3 and 6 , and duration between 0.5 and 


\begin{tabular}{|c|c|c|}
\hline Treatment details & Visual act & uity \\
\hline $\begin{array}{l}\text { Diathermy (ma/sec.) } \\
\text { *Photocoagulation No.; } \\
\text { Intensity (I);Size (S); } \\
\text { Duration (sec.) (D) }\end{array}$ & $\begin{array}{l}\text { Before } \\
\text { treatment }\end{array}$ & $\begin{array}{l}\text { After } \\
\text { treatment } \\
\text { (follow-up) }\end{array}$ \\
\hline
\end{tabular}

PC: 84,5 (I),
3 (S) 1.5 (D)
PC: 25,5 (I),
4.5 (S), 1.5 (D)
PC: $87,7.5$ (I),
3 (S), 1.5 (D)
PC: 60.7 (I),
4.5 (S), 3 (D)

PC: $54,7.5$ (I), $6 / 6$ 4.5 (S), 1.5 (D)
PC: $36,7.5$ (I),
4.5 (S), 1.5 (D) 4.5 (S), 1.5 (D)
PC: $80,9 \cdot 5$ (I), 3 (S), 2 (D)
(D) Large arteriole PC: 21,7 (I), 4.5 (S), 3 (D) PC: 48,7 (I) 3 (S), $2 \cdot 5$ (D) PC: 94, 10 (I),

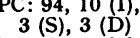
3 (S), $3(\mathrm{D})$
Large arteriole PC: 149,9 (I) 3 (S), 3 (D) Large arteriole

PC: $140,9(\mathrm{I})$
$4 \cdot 5(\mathrm{~S}), 3(\mathrm{D})$
PC: 115,10 (I),
$4 \cdot 5$ (S), 3 (D)
Asteroid hyalitis

PC: 68, 10 (I),
3 (S), 3 (D)
Pterygium

PC: 66,3 (I),
$4 \cdot 5$ (S), 1.5 (D)
Inadequate intensity
Second 10 (I)
treatment:
PC: 139, 10 (I),
3 (S), 2.5 (D)

$6 / 6$

$6 / 6$

$6 / 9$

$6 / 9$

(8 wks)

$6 / 6$

$6 / 6$

(6 wks)

$6 / 6$

$6 / 6$

(8 wks)
Post-treatment

assessment

Fluorescein leakage

Retinoschisis (S)

Retinal detachmen

(RD)

Leakage -

Leakage -

Leakage -

Leakage -

Leakage -

Leakage -

Leakage -

Leakage -

Leakage -

Leakage -

Leakage -

Single localized vitreous haemorrhage after second treatment

2 retinal haemorrhages in

coagulation burn

2 retinal haemorrhages in coagulation

burn

Partial III nerve palsy, 4 days

Partial III nerve palsy, 5 days

Leakage -

S +

Leakage -

(6 RPs)

Leakage +

(2 untreated RPs

Leakage -

(3 RPs)

Leakage +

(1 untreated $R P_{\text {, due }}$ to pterygium)

First: Leakage + (1 RP)

Second: Leakage -

First: Leakage $+\quad 1$ subretinal haemorrhage in

(1 RP) coagulation burn

2 sec., was applied directly to the wall of the feeder arterioles at a point 0.5 disc diameter posterior to the lesion. The coagulated retina in relation to the vessel underwent the usual grey followed by white reaction after which the retinal vessel went into spasm and closed down. It was important that the burn be prolonged slightly after vessel spasm had occurred and not discontinued as spasm was occurring. The vessel in question was then carefully watched with the coagulator light for 2 to 3 minutes. If the spasm disappeared and the vessel re-opened, a pulsating cattle-truck appearance was easily seen against the white background of the burn, in which case the power of the beam was increased slightly and a similar burn applied adjacent and posterior to the first burn and directly to the vessel wall. A length of untreated vessel wall was always left between the area of coagulated vessel and 
$(a)$

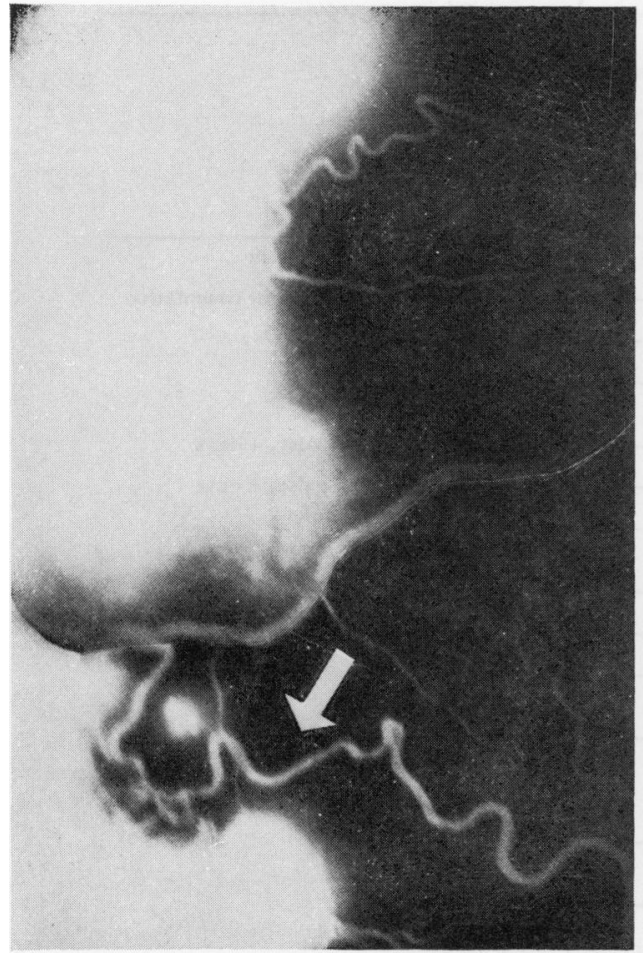

(b)

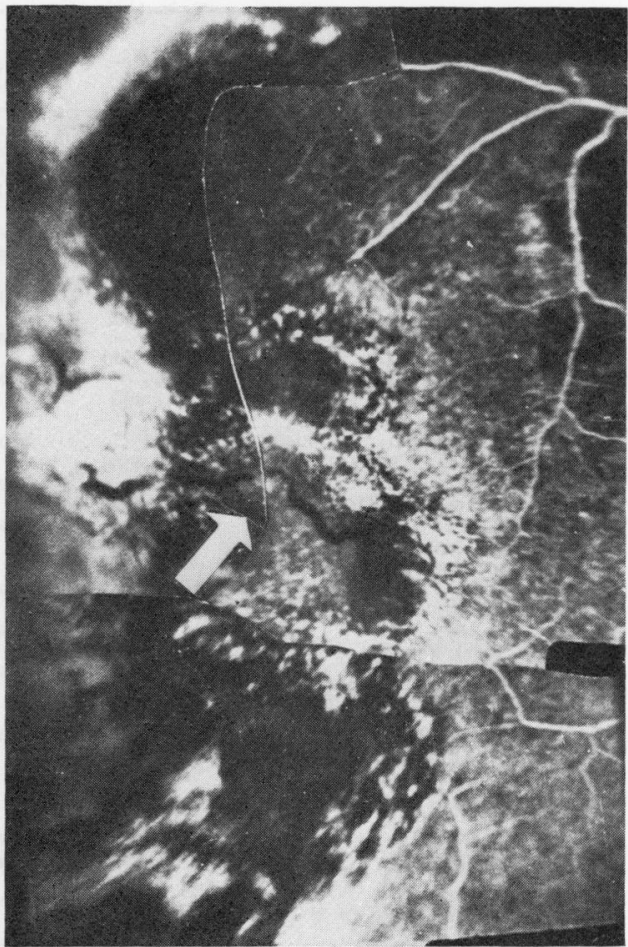

FIG. $2(a, b)$ Case 12. Fluorescein angiograms of elevated sea-fan lesion before and after treatment. Complete occlusion of large feeding vessels $(\rightarrow)$ and absence of dye leakage in $(b)$ demonstrate a 'therapeutic cure'

the RP lesion to allow re-treatment if necessary at a later time. Care was taken to avoid coagulation of any veins draining from the lesion. When all the feeding arterioles to a single area of RP had been coagulated in this way, the power of the beam was reduced by $I$ or 2 units and the area of new vessels was directly coagulated. All areas of RP in each eye were treated in one treatment session, except in three patients with extensive bilateral involvement in whom limited treatments were spread over 2 months to minimize retinal reaction in the treated eye. The coagulator was normally used with the maximum diaphragm opening except in extensive peripheral lesions when it had to be reduced to avoid iris heating. Also, in the treated eye, areas of suspected hypoxic retina on the border between the ischaemic and healthy areas, in which early new vessel formation and arterio-venous shunts were developing, were treated by photocoagulation in order to render them completely ischaemic.

\section{Diathermy technique}

Using standard retinal detachment techniques, the areas of RP and the major feeder vessels were localized by indentation with accurately placed marker sutures on the scleral surface. A disposable spatulated atraumatic needle was used to insert the marking stitch and care was taken to ensure that the needle did not penetrate too deeply and produce a retinal tear. A lamellar scleral resection was performed over the marked area, and this was extended, if possible, to the point where the feeder vessels regained the normal retinal level. Diathermy was applied to the whole of the resected area and was continued until a white reaction appeared. An adequate burn was achieved by an average current of $70 \mathrm{~mA}$ for $6 \mathrm{sec}$. If the lesion was pulled forwards from the retinal surface with schisis extending beyond the equator posteriorly, the feeder arterioles were photocoagulated before they left the retinal surface and diathermy was subsequently applied directly to the lesion (Plate II $b$; Fig. $2 b$ ). Diathermy burns were not applied more than I disc diameter posterior to the equator, and areas behind this were left untreated once the feeder arteriole was closed. The area treated by diathermy was kept to a minimum, photocoagulation being used as a supplementary therapy when required. 
Postoperatively, all patients were given topical steroids to control the mild anterior uveitis which usually followed, and in two patients systemic steroids in high doses were required for short periods to control vitreous clouding from mild posterior uveitis.

\section{Anaesthetic and other procedures}

Fourteen of $3^{1}$ patients receiving photocoagulation were treated on an out-patient basis and the remainder were admitted for periods not exceeding 4 postoperative days. In general, hospital admission was reserved for patients with complex lesions or those who for social reasons would not be able to have complete rest in bed at home.

All patients treated by diathermy were admitted to hospital.

Local anaesthesia was used in all cases because of the risks of general anaesthesia in sickle cell disease (Howells, Huntsman, Boys, and Mahmood, 1972). A facial block and a retrobulbar injection of 2 per cent. Xylocaine without adrenaline was used in all procedures, the resulting proptosis aiding accessibility. Because of the long duration of diathermy procedures, continuous intravenous diazepam and pethidine were also administered.

\section{Results}

PHOTOGOAGULATION (Table II)

Table II Short-term results of photocoagulation therapy

\begin{tabular}{|c|c|c|c|c|c|c|c|c|c|c|}
\hline \multirow{3}{*}{ Treatment } & \multirow{3}{*}{$\begin{array}{l}\text { No. of } \\
\text { patients }\end{array}$} & \multirow{3}{*}{$\begin{array}{l}\text { No. of } \\
R P \\
\text { lesions }\end{array}$} & \multicolumn{8}{|c|}{$\ddagger$ Results (days after initial therapy) } \\
\hline & & & \multicolumn{2}{|l|}{4} & \multicolumn{2}{|l|}{28} & \multicolumn{2}{|l|}{42} & \multicolumn{2}{|l|}{56} \\
\hline & & & Occluded & Patent & Occluded & Patent & Occluded & Patent & Occluded & Patent \\
\hline Single & 29 & 100 & 95 & $5^{*}$ & 95 & $5^{*}$ & 95 & $5 *$ & 95 & $5^{*}$ \\
\hline $\begin{array}{r}\text { same area } \\
\text { Repeated to }\end{array}$ & 4 & 19 & 15 & 4 & 15 & 4 & 15 & $4 \dagger$ & 18 & 1 \\
\hline different areas & 3 & 18 & 12 & 6 & 12 & $6+$ & 18 & $\mathbf{0}$ & 18 & 0 \\
\hline Total & 36 & 137 & 122 & 15 & 122 & 15 & 128 & 9 & 131 & 6 \\
\hline
\end{tabular}

* Includes three lesions in inaccessible areas and one missed lesion

All patients received initial treatment on Day 0

+ Indicates time of additional treatments

$\ddagger$ Results of therapy depicted as RP lesions (occluded or patent)

This was used alone in $3 \mathrm{I}$ patients (2 I male; Io female) and in association with diathermy in five (three male; two female). In a total of 45 separate therapy sessions, 137 lesions of RP were treated by occluding 230 feeding retinal arterioles and coagulating an average of $128^{\circ}$ of arc of the peripheral retinal circumference in each eye. In 26 patients, a total of 85 lesions of RP received single treatments which led to occlusion in 83 lesions within 4 days. Subsequent spontaneous closure in one other lesion took several weeks to occur (Figs $3 a, b$ ). The patient with the remaining patent RP lesion failed to attend for further therapy. In three other patients, therapy was given in repeated limited procedures and all eighteen lesions of RP were successfully occluded. In four patients, repeated coagulation was required to four previously treated RP lesions several weeks later and vascular occlusion was achieved in three out of four RP lesions, with marked reduction of blood supply in the fourth. These patients were all treated at the beginning of the study when the techniques of vessel closure and the power of the photocoagulator were being assessed. In two patients the extreme peripheral site of the lesions or the presence of opacities in the ocular media allowed successful occlusion in only six out of nine lesions. In one patient one area was inadvertently missed.

The photocoagulator was capable of closing moderately-sized peripheral retinal arterioles, and the settings most frequently used were intensity 7 , burn size 4.5 , and duration 2 
(a)

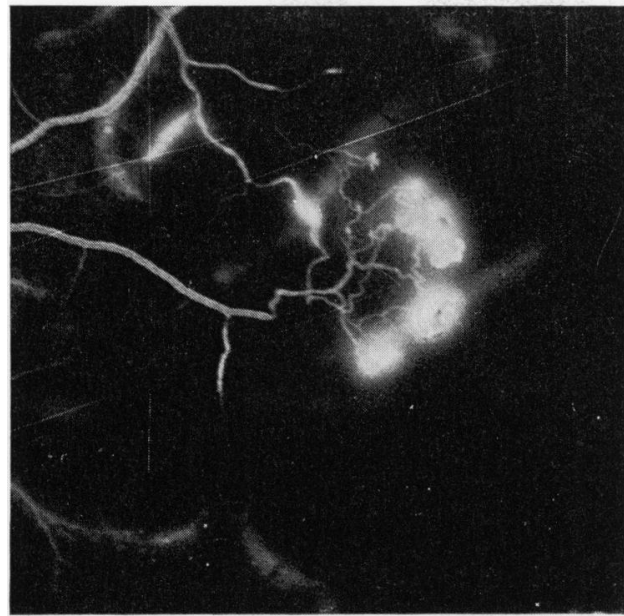

(b)

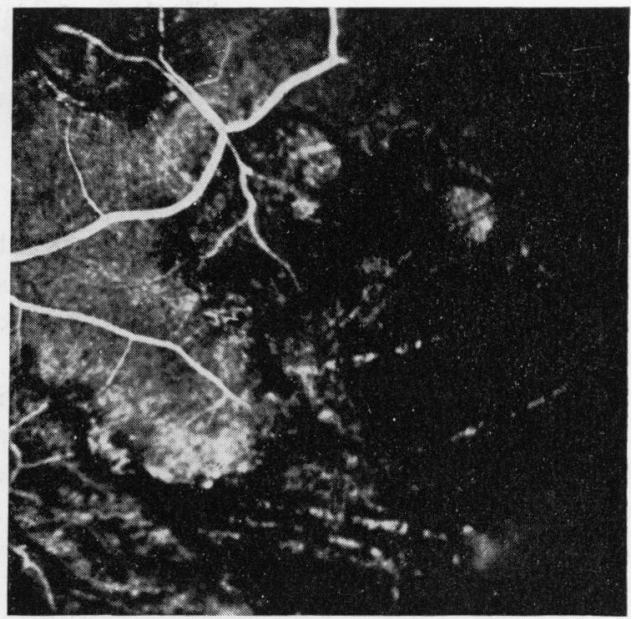

FI G. $3(a, b)$ Case 36 . Fluorescein angiograms of large flat RP lesion after coagulation demonstrate persistent patency of a single feeding arteriole and leakage of dye in a segment of the lesion 4 weeks after treatment (a). Spontaneous gradual closure of arteriole within 7 weeks and absent dye leakage (b) resulted in atrophy of lesion and a 'therapeutic cure'

sec. Increased intensity 9 or Io was required in five patients with opacities in the media and in six patients with large retinal arterioles (Plates III $a, b$; Figs $4 a, b$ ). In general, burn sizes between 4.5 and 6 were used, but smaller burns were required when artery and vein ran closely together. Burn duration varied from $1 \cdot 5$ to $3 \mathrm{sec}$. depending on the time required to induce vessel spasm.

\section{Complications}

Immediate and short-term complications of photocoagulation were few. Inadvertent coagulation of a large draining vein caused an immediate localized retinal and preretinal haemorrhage in one patient (Plate IV); this did not affect vision and resolved over 6 weeks. In some patients, fluorescein angiograms revealed a slight leakage of dye through the arteriolar wall at the burn site (Figs $5 a$, and $b$ ), and in five patients, eight round retinal haemorrhages appeared localized to areas of a single burn (Plate V). The haemorrhages resolved rapidly and further angiography revealed that fluorescein leakage subsequently ceased. One patient who returned to work within 2 days contrary to instructions, developed a vitreous haemorrhage, but this remained localized to the area of therapy, did not affect vision, and resolved within 3 weeks. In three patients local anaesthesia produced a partial III nerve palsy which slowly resolved. This complication was not seen after reduction of the volume of retrobulbar anaesthetic from 6 to $4 \mathrm{ml}$.

\section{DIATHERMY}

This was used alone in eight patients and in association with photocoagulation in five. It was applied to 29 lesions of RP and fifty feeding arterioles, to an average of $87^{\circ}$ of arc of the peripheral retinal circumference in each eye. In all patients, diathermy achieved a therapeutic cure by sealing off the feeder vessels to the RP areas, and in some cases it had a direct coagulative effect on the leaking vessels in the bed of the sea-fan. In eleven patients, diathermy also resulted in absorption of the intraretinal and subretinal fluid, the retina settling into a pigmented area with a firm chorio-retinal adhesion. The condensed vitreous usually remained in its preretinal location. In two patients, the extreme anterior location 
FI G. 4 (a) Case 36. Fluorescein angiogram of sea-fan lesion, demonstrating large feeder arterioles and profuse dye leakage before photocoagulation (cf Plate III $a, b$ )

FIG. $4($ b $)$ Case 36 . Fluorescein angiogram after photocoagulation, demonstrating complete closure of larger feeder arterioles and absent dye leakage leading to a 'therapeutic cure'

of the RP lesions treated by diathermy prevented the associated schisis from disappearing completely, but there was an improvement in one.

\section{Complications}

The most serious complication encountered was that of anterior segment ischaemia which occurred in one patient who had complete involvement of the periphery with RP. Diathermy was applied to three areas of traction retinal detachment in three quadrants up to $180^{\circ}$ of arc of the retinal circumference, and care was taken to avoid areas of sclera overlying the long posterior ciliary vessels. The extraocular muscles were not detached from the eye in the course of surgery. An anterior uveitis occurred in all patients, but this resolved over 4 weeks with the use of topical mydriatics and steroids. In two patients, a posterior uveitis necessitated systemic steroid therapy. Small round preretinal haemorrhages related directly to retinal arterioles occurred immediately posterior to areas of diathermy in three patients and in unrelated areas in three others (Plate VI). Visual acuity was unaffected by the treatment in twelve cases and was reduced in only one patient, who developed a 
(a)

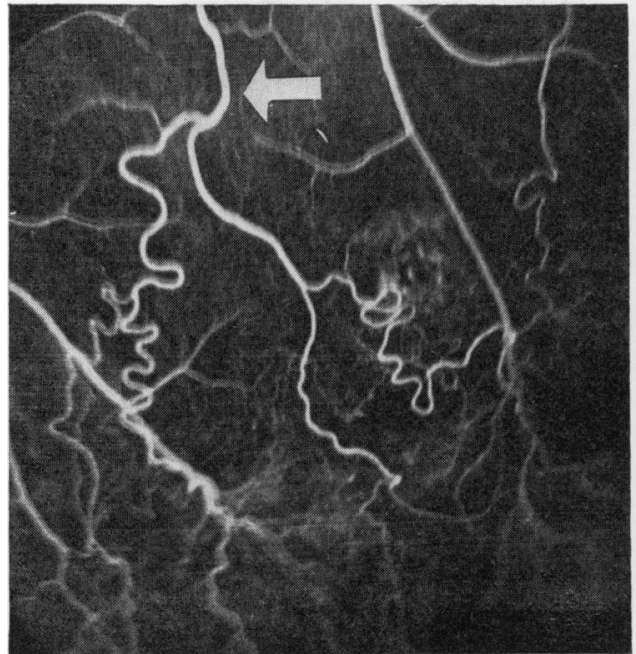

(b)

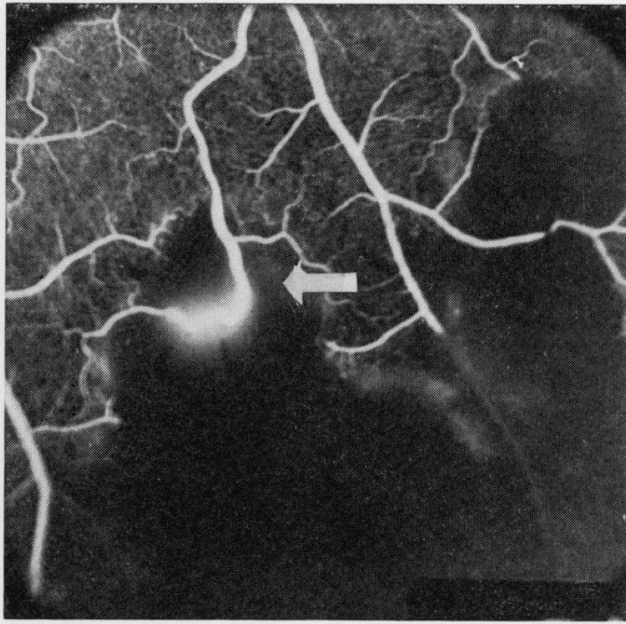

FI G. $5(a, b)$ Case 42 . Fluorescein angiogram demonstrating dye leakage through treated necrotic arteriolar wall with successful occlusion of feeder arterioles $(\rightarrow)$ leading to a therapeutic cure

posterior ciliary artery occlusion 3 months after treatment of a $90^{\circ}$ area of RP. In the patient with anterior segment ischaemia, visual acuity had remained normal for 5 months after therapy when this report was submitted.

\section{Discussion}

Retinitis proliferans was found in nearly one-third of adults with SC disease (Condon and Serjeant, I972c) and was also common in childhood, occurring as early as 6 years of age (Condon and Serjeant, 1974). The high incidence of RP amongst adults with the relative infrequency of blindness suggests that these lesions have a considerable capacity for spontaneous regression. However, despite this tendency to natural regression, the complications of RP and their sequelae in some patients justify attempts at therapeutic occlusion, although the effects of this approach on the natural history of the retinal disease remains at present unknown. It was for this reason that one eye was left as a control.

The three main therapeutic approaches have included diathermy and Xenon arc and Argon laser photocoagulation. In preliminary studies before the present series was undertaken, photocoagulation applied directly to two elevated RP lesions appeared to cause an increase in vitreo-retinal traction and subsequent retinal detachment in two patients. This complication has been reported after photocoagulation in proliferative diabetic retinopathy if vitreo-retinal traction was present before treatment (Patz, I973). Also, Goldberg (I97 I b) reported a 5.2 per cent. incidence of photocoagulation-induced vitreous haemorrhage in 58 treatment episodes from RP lesions located pre-retinally. The increased risks of haemorrhage and detachment in RP lesions with vitreo-retinal traction led to the selection of diathermy, since this provided a firmer chorio-retinal adhesion and required less heating of the vitreous. Diathermy also had the added advantage that it coagulated any new vessels near the retinal surface.

The instrument used for photocoagulation was the O'Malley Log II; this has the advantage of being portable and can be used from standard power supplies. The maximum setting of ro corresponds to "red one" or "green three" on the West German Zeiss instrument (O'Malley, I972) and in only one case did the portable instrument have insufficient 
power to occlude an area of RP. Other advantages of the portable instrument were the free mobility and the low inertia of the delivery system and fingertip rotation of the mirror, both of which facilitated coagulation of the peripheral retina and permitted the surgeon to remain seated throughout the procedure.

Photocoagulation to RP lesions can be directed at the new vessels themselves, provided they are on the retinal surface, or at the feeding arterioles where they lie on the retinal surface. In preliminary studies before this report, vitreous haemorrhage with resultant impairment of visual acuity occurred in two patients in whom surface RP lesions alone were directly coagulated. Goldberg ( $197 \mathrm{Ib}$ ) has also shown that photocoagulation-induced vitreous haemorrhage is more likely to occur from new vessels elevated from the retinal surface rather than from the directly coagulated feeder arterioles themselves. The technique of Archer, Krill, and Newell (1970) of direct coagulation to the vessel wall was used in this study. This was selected because of the success of this coagulator in occluding healthy cat retinal arterioles by this technique (Hill and Young, 1974) and in spite of the dangers of haemorrhage from the necrotic wall after coagulation in other conditions such as Eales's disease, diabetic retinopathy, and retinal angiomatosis (Meyer-Schwickerath, 1972). In this report, the technique of closing the feeding arterioles before directly coagulating the new vessels was associated with minor vitreous haemorrhage in only one patient in a total of 230 treatments. In five coagulation burns in five patients, there were small localized retinal haemorrhages into the coagulated area, and in eight coagulation burns slight fluorescein leakage was seen to occur through the vessel wall in the coagulated area. It is assumed that the low haemorrhage rate in this series is due to the relative absence of pathological disease in the feeder arterioles and to their ability to maintain a significant spastic state when coagulated in this way.

Complications from both procedures were minimal, the only serious problem being anterior segment ischaemia which occurred in a patient treated by diathermy. This has been reported after standard retinal detachment procedures in sickle cell disease (Ryan and Goldberg, I971) and is considered to be associated with removal of the extraocular muscles from their insertions, damage to the long posterior ciliary vessels, and the use of diathermy or cryopexy (Eagle, Yanoff, and Morse, I973). However, in nine cases of this series, diathermy was applied over one or other long posterior ciliary vessels without subsequent ischaemia, and in one patient who did develop this complication no extraocular muscles were removed from the globe and diathermy directly over the course of the long posterior ciliary vessels was avoided. A difference between this and the other cases was the relatively larger area of retina treated and the greater extent of retinopathy before treatment. Three other patients had diathermy applied to more than $90^{\circ}$ of the retinal circumference without serious complications, but it is considered advisable to restrict diathermy to $100^{\circ}$ of the total retinal circumference and to supplement it by photocoagulation of the feeder arterioles.

The effect of obliterating RP lesions in one retinal quadrant on the subsequent formation of similar lesions in other quadrants is unknown. In the first seven cases of this series, which were treated by diathermy and followed for a period of 2 years (Table I), no new lesions were observed. It is possible that the re-direction of blood from successfully occluded seafans may increase perfusion to other relatively hypoxic areas and prevent RP lesions from developing.

The patients treated by photocoagulation were observed for an 8-week period during which re-canalization of successfully occluded arterioles did not occur. Nor did any form of maculopathy arise from the coagulation of large areas of the peripheral retina. Further 
longitudinal observations, which will be necessary to assess the long-term effects of these methods of treatment, are in progress.

\section{Summary}

The O'Malley Log II portable photocoagulator was used to treat the worse eye of 36 patients with proliferative sickle cell retinopathy flat on the retinal surface. The technique involved direct coagulation of the feeder arterioles before treatment of the new vessels themselves. The photocoagulator was most successful in treating the lesions and I 3 I of I 37 retinitis proliferans (RP) lesions were occluded. In only one RP lesion did the photocoagulator have insufficient power to occlude the lesion. Vitreous haemorrhage occurred in only one patient, and small round localized retinal haemorrhages in five.

The Manchester portable diathermy machine successfully occluded all 29 raised RP lesions and fifty feeder arterioles in thirteen patients. Anterior segment ischaemia occurred in only one patient who had advanced traction retinopathy involving $360^{\circ}$ of arc of the retinal circumference before treatment. No recurrence of retinopathy over a 2-year followup period was seen in seven patients, and in the remainder there was no recurrence in the 2 months after treatment.

We wish to thank Prof. David W. Hill for his advice and guidance in the management of some of the cases and for assistance with the manuscript.

We are extremely grateful to Mr. Hughes de Laforcade, of Clinitex, Inc., ${ }_{363}$, Rantoul St., Beverly, Mass. orgi5, U.S.A., the manufacturers of the O'Malley Log II Photocoagulator, and to Mr. Michael Ham of Keelers, London, U.K. agents for the instrument, both of whom were instrumental in loaning us a machine for this study. The cost of the colour plate was also most generously given jointly by both firms. Our grateful thanks are also due to Miss Philpotts, Chief Photographer, Photographic Department, St. James' Hospital, Balham, S.W.12, for her help with the illustrations, to Mr. Albert Lockhart, F.R.C.S., Ophthalmic Surgeon, to sister Anderson and staff, University Hospital of the West Indies, in whose department most of the work was carried out, and to Miss Margaret Keehan for secretarial assistance.

\section{References}

APPle, D. J., Goldberg, M.F., and wyhinny, g. (1973) Amer. J. Ophthal., 75, 595

ARGHeR, D., KRILl, A.E., and NeWell, F. W. (1970) Trans. ophthal. Soc. U.K., 90, 677

condon, P. I., and serjeant, g. R. (ig72a) Amer. 7. Ophthal., 73, 533



eagle, R. C., yanoff, M., and morse, P. H. (i973) Amer. 7. Ophthal., 75, 426

Goldberg, M. F. (I97Ia) Arch. Ophthal. (Chicago), 85, 428

- (1971 Ib) Trans. Amer. Acad. Ophthal. Otolarvg., 75, 532 and Acacio, I. (1973) Arch. Ophthal. (Chicago), 90, 35

hannon, J. F. (1956) Amer. J. Ophthal., 42, 707

hoWells, T. H., huntsman, R. G., Boys, J. E., and mahmood, A. (i972) Brit. $\mathcal{J}$ Anaesth., 44, 975

HILL, D. W., and young, s. (1974) Exp. Eye Res., (in press)

кEITH, c. G. (I968) Brit. J. Ophthal., 52, 862

MEYER-SCHWICKERATH, G. (I972) Personal communication

O'MAlley, P. (1972) Personal communication

PATz, A. (1973) Highlights Ophthal. 1972-73 series (Int. ed.), 14, 5

RYAN, s. J., and Goldberg, M.F. (I97I) Amer. J. Ophthal., 72, 35

SERJEANT, G. R., and SERJEANt, B. E. (I972) Brit. F. Haemat., 23, 205 